\title{
Zur Naturgeschichte des Girlitz, Fringilla Serinus Lin. Von
}

Alex. v. Homejer.

Sechs Jahre in Frankfurt a. M., - in einer Gegend, in der der Girlitz sehr häufig, vielleicht von ganz Deutschland am häufigsten ist, dazu manche interessante Notiz in den ornithologischen Zeitschriften über seine noch nicht genügend bekannte Verbreitung, wie Aufklärung seiner Naturgeschichte selbst, - veranlassten mich, dem Girlitz meine Aufmerksamkeit zu widmen. Ganz besonders willkommen war mir der durch Herrn Dr. Julius Hoffmann gelieferte Aufsatz*), welcher mir bei meinen Beobachtungen vielfach als Richtschnur diente, indem er auf das Interessante der Lebensweise aufmerksam machte; möge mir demnach erlaubt sein, auch hier mich an diese, in jeder Beziehung vorzügliche Arbeit**) zu halten und einige bezügliche Bemerkungen folgen zu lassen, wobei gleich gesagt sein mag, dass Vieles übereinstimmend ist und demnach nur als eine Bestätigung gelten mag, und nur Weniges abweicht, worüber von Anderen entschieden werden möge. Zur Sache also!

Julius $H$ off mann sagt

1. Ueber die Ankunft: dass einzelne Vögel Anfang April, alle erst in der Mitte dieses Monats da seien.

Meine Beobachtungen sind folgende:

Im Jahre 1854 sangen am 1. April sehr viele Girlitze in der Stadtpromenade von Frankfurt a. M., woraus hervorgeht, dass die ersten Ankömmlinge gewiss 8 Tage früher einpassirt seien, also am 23. März, einer Zeit, welche genau mit der Ankunft in den folgenden Jahren übereinstimmt.

1855 nämlich sang der erste Girlitz ebenda schon am 19. März, 56 am 24., 57 am 23. einige, 58 der erste am 24, viele am 2. April, 59 der erste sogar schon am 13. viele am 19. März. Im Jahr 1960 verliess ich Frankfurt am 9. März, und war bis dahin der Girlitz noch nicht angekommen; den ersten sah ich am 5. April in Baden-Baden am Alten-Schloss, den zweiten am 15. April ebenda am Neuen-Schloss.

Demnach dürfte für Frankfurt a. M. der Frühjahrszug etwas

*) Naumannia II. 3. p. 58-64.

**) Je mehr und länger ich mich mit dem Vögelchen beschäftigte, desto mehr lernte ich die Arbeit würdigen.

Journ. f. Ornith., X. Jahrg., Nr. 56, März 1862. 
früher als für Stuttgart fallen, etwa für die ersten Ankömmlinge am 20. bis 24. März, für den Hauptzug am 1. April.

In Betreff des Herbstzuges bin ich ein schlechter Beobachter, wenigstens für die Details, und weiss ich desshalb auch nichts Näheres anzugeben, als dass im August die Girlitze mit ihren Jungen flugweise auf die Felder gehen und Ende September oder Mitte Oktober uns verlassen, während einzelne noch Anfangs November zu sehen sind.-

Am Mittel-Rhein überwintert der Girlitz nicht, und wurden mir nur 2 A usnahmefälle bei Mainz bekannt, während ich es bei Frankfurt nicht beobachtete. Das erste Mal war es im Febr. 54, als ein laut lockendes Männchen an mir vorbeiflog, das 2. Mal im strengen Winter 60 bis $\mathbf{6 1}$, dass sich drei Vögel bei hohem Schnee dicht vor meinem Fenster auf einem Gartenstück des alten Kästerich sehen liessen, ohne sich den Hänflingen und Stieglitzen intim anzuschliessen, denn oft genug waren die Vögel einzeln oder unter sich; einige Tage darauf sass ein Trupp von 8 Stück vor meinem Fenster, welcher acht Tage lang blieb und dann der rauhen Witterung halber weiterzog.

2. Ueber sein Treiben gleich nach der Ankunft: Die ersten sind stets Männchen, und diese machen sich sogleich durch ihren Gesang, den sie von den Baumspitzen ertönen lassen, sowie durch ihr unruhiges Treiben sehr bemerkbar.

Wenn die Witterung schön, d. h. hell, sonnig und milde ist, so hat es vollkommen seine Richtigkeit mit dem munteren Treiben des lebhaften Vögelchens und mit dem schnellen Sichbemerkbarmachen; denn die singenden Männchen schwirren immerzu oder treiben sich mit einem zizizi händel- und eifersüchtig aus dem kleinen Revier ihres zukünftigen Nistplatzes. Ist es aber kalt und unrüstig, namentlich windig und regnerisch dabei, oder treibt gar Schnee, - eine Witterung wie wir sie Ende März und Anfang April so oft haben, - so macht unser Vögelchen ein ganz anderes Gesicht. Es hält sich niedrig, um Schutz gegen die Witterung zu finden, und lockt nur hier und da leise und verstohlen aus einem Strauch heraus, oder trippelt der Nahrung halber auf der Erde neben einem Meldenstrauch, - aber immer ruhig, ohne bei seiner schlechten Laune viel Wesen's und Lärm zu machen. So kann es bei anhaltend ungünstiger Witterung kommen, dass schon viele Girlitze vorhanden sind, ohne dass man 
viel von ihnen sieht, und dass dann beim ersten Sonnenblick er in Unzahl von allen hohen Bäumen herabsingt.

Das eigenthümliche grünlingsartige Fliegen, was immer von heftigem Singen begleitet ist, geschieht erst Mitte April; oder wenn dieser Monat kalt ist, erst von Anfang Mai ab, und scheint mir ein Balzen zu sein, herbeigeführt durch regen Geschlechtstrieb.

3. Der Gesang hat die meiste Aehnlichkeit mit dem des Accentor modularis.

Hoffmann's Vergleich ist vorzüglich; der Gesang ist durchaus dem einer Heckenbrunelle (Accentor modularis) so ähnlich, dass er mit ihm verwechselt werden kann, wie es wenigstens mir am 1. April 1854 erging, als ich nach Frankfurt kommend, den Girlitzgesang noch nicht kannte. Dieser Irrthum dauerte jedoch nur wenige Minuten, dann wusste ich mir den „eigenthümlichen Brunellengesang" sofort zu deuten; Hoffmann weiss dies ,eigenthümlich" ganz prächtig mit seinem „Finkenschnabel" zu charakterisiren, d. h. das Klangvolle der Brunelle fort, und dafür S- und R-Töne, wodurch der Gesang hart wird, und nun noch das Tempo der Carriere hinzu, wodurch fast ein Schwirren erzielt wird. - Dem Gesange des Goldhähnchens (Regulus), wie Baldamus will, ist der Gesang allerdings auch ähnlich, doch gefällt mir Hoffmann's Vergleich ungleich besser, wenigstens würde es mir nie passiren, Girlitz- und Goldhähnchengesang mit einander zu verwechseln; der Girlitzgesang entbehrt einmal jeglichen sanften Characters. Aehnlichkeitsbeziehungen erster Linie sind ferner noch mit dem Gesange der Fringilla citrinella vorhanden, welcher zwischen Stieglitz- und Girlitzgesang "genau mitteninne" steht.*)

Der Vergleich des Gesanges mit dem Kanarienvogel will mir durchaus nicht gefallen; mit diesem hat der Girlitz nur die piependen Töne unmittelbar vor der Begattung wie das „äd“ gemein, welch' letzterer Ton von beiden Vögeln in Mitten des Singens während einer kleinen Pause so gern gegeben wird und bei Beiden mit einem Seitwärtswenden des hinteren Körpertheiles begleitet ist, welches beim Kanarienvogel langsam, bei Letzterem, bedingt durch die grosse Lebhaftigkeit, sehr oft und sehr schnell mit hängenden Flügeln und gehobenem Schwanze geschieht.

Sehr bezeichnend für den Gesang ist übrigens der bayrische Name unseres Vögelchens „Hirngritterl“, wie es uns Pfarrer Jäckel

*) Siehe darüber dieses Journal VIII. S. 372 und IX. S. 71. 
in der Naumannia III., S. 394, mittheilt, indem der ganze Character des Schwirrgesanges mit seinen R-Tönen darin enthalten ist.

Was den Gesang in der Stube anbetrifft, so hat er seine Liebhaber; Herr Eduard Kuhlmann*) schwärmt sogar für ihn; dieser hielt stets 1-3 Vögel "des Gesanges halber" im Käfig, und charakterisirte mir diesen 1852 in Mainz durch die Worte: ähnlich schwirrend und stotternd wie der Grauammer, aber der Ton klangvoller und der Gesang abwechselnder und länger.

Mir ist der Gesang im Freien seiner Eigenthümlichkeit halber stets willkommen, im Zimmer jedoch kann ich ihn nicht leiden, und so wird es gewiss einem Jeden ergehen, dessen $\mathrm{Ohr}$ ein Wenig durch die sanften Töne der Sylvien verwöhnt ist, ja ich sage, dass die Härte des Girlitzgesanges sogar nicht mit andern Finkengesängen harmonirt und somit auf die Dauer stört.

4. Sehr eigenthümlich ist die Vorliebe des Girlitz für bestimmte Orte. In der nächsten Nähe von Stuttgart ist er ziemlich gemein, aber über eine Stunde ringsum ist gewiss kein einziger anzutreffen.

Dies ist sehr richtig. Der Girlitz kann an einem Ort sehr häufig und 4 Meilen davon eine Seltenheit sein, wenn auch dem Anscheine nach die Oertlichkeitsverhältnisse die nämlichen sind; dies gilt (oder galt, wie wir gleich sehen werden), z. B. von Frankfurt a. M. und Mainz. Das Warum jedoch vermag man nicht zu deuten, und darin liegt eben das Räthselhafte, was noch vermehrt wird, wenn dann an einem solchen nicht besuchten Ort der Girlitz sich plötzlich sehr zahlreich einstellt, ohne dass die Verhältnisse günstiger für ihn wurden (s. Mainz und den Nachtrag über Hanau), oder auch plötzlich verschwindet (s. Rastatt).

Bei Frankfurt a. M. also und ganz speciell in der dortigen Stadtpromenade ist der Girlitz ein so häufiger Vogel, dass man kaum dem Sperlinge**) das Vorrecht geben kann. Man findet ihn ferner bei sämmtlichen benachbarten Dörfern und den dazwischen liegenden Obstbaumpflanzungen, wenn auch nicht so häufig, wie in der Promenade selbst, welche als eigentlicher Lieblingsaufenthalt der ganzen Gemarkung zu betrachten ist.

Als ich 1852 zu Mainz in Garnison stand, war der Girlitz daselbst eine grosse Seltenheit; im Gartenfelde, einem ausgedehnten Baum- und Buschgartenstück, lebten damals kaum 2 Paare;

*) Grossherzoglich Hessischer Oberzollamts-Revisor und Freund der Vögel.

**) Der allerdings gerade nicht sehr zahlreich ist. 
später etwa 1858 vernahm ich durch Herrn Conservator Nicolaus, dass der Girlitz etwas häufiger würde, und im Frühlinge 1860 hörte ich ihn daselbst überall in so unglaublicher Menge singen, dass ich die Zahl eben so gut auf 100, wie nur auf 50 angeben kann.

In Hanau ist er jetzt häufig. (S. Nachtrag.)

Bei Offenbach überall, jedoch nicht zahlreich.

Bei Trier kam er $\mathbf{5 3}$ nicht vor.

Bei Durlach im Rheingau war der Girlitz vor einigen Jahren nach Herrn Förster Diess ziemlich häufig, jetzt nicht.

Bei Rastatt sah ich ihn nicht, obwohl für treffliche Oertlichkeit gesorgt zu sein scheint (Schloss Favorite). Durch den alten Vogelfänger Schwan, der unsern Vogel ganz genau kennt, und für den ich überhaupt einstehe, erfuhr ich, dass der Girlitz daselbst früher sehr häufig gewesen, dass sein Vorkommen jetzt aber so vereinzelt wäre, dass er nur in einzelnen Jahren einige Junge während des Herbstes auf den Salatstauden gefangen hätte.

Bei Baden-Baden beobachtete ich im April 1859 zwei Paare, und endlich am 16. Juni und die folgenden Tage ebenda viele singende Männchen. Da diese nun vorhin nicht daselbst gewesen waren, es überdies für die Zugzeit bedeutend zu spät war, so glaube ich, dass es Brutvögel des Schwarzwaldes waren, welche nun mit ihren Jungen in's Thal hinabstiegen. -

5. Er ist nie auf der Höhe, sondern nur im Thal, oder auf ziemlich ebenem, tief liegendem Terrain zu suchen.

Im Allgemeinen stimmt dieses allerdings, namentlich für Frankfurt, dem Lieblingsaufenthalte, aber nicht für Baden-Baden, Gernsbach, Ebersteinschloss, den Fremersbergen, dem Merkur, kurz den westlichen Schwarzwaldpartien. Hier in fast geschlossenen 80jährigen Weisstannen-Waldungen, welche mit Buchen durchwachsen sind, trifft man den Girlitz, wenn auch gerade nicht häufig, so doch fast überall zu $1-3$ Pärchen, namentlich auf den gegen Süden liegenden Bergabhängen.

Ich gestehe, dass einerseits bei der Aehnlichkeit des Girlitzund Citronenzeisig-Gesanges, wie andererseits wegen Hoffmann's definitiven Urtheils „er ist nie auf der Höhe“, ich im Schwarzwalde bei einem mir sonst doch so bekanntem Vogel ganz irre wurde und lange nicht wusste, wen ich denn eigentlich vor mir hätte, bis ich denn endlich sicher beobachtete, dass dies hier der 
Girlitz sei, während der Citronenzeisig im eigentlich hohen Schwarzwald und dessen östlich nach Würtemberg zu liegenden Abhängen vorkommt.

6. Er hat für den Birnbaum eine ganz besondere Vorliebe, auch für den Zwetschenbaum; auf einem Apfel-oder Kirschbaum fand ich noch kein Nest, und im Nadelholz, wie Herr Heuglin im 3 ten Heft der Naumannia vermuthet, möchte er wohl nie brüten.

Bei Frankfurt a. M. nistet der Girlitz vorzugsweise a u $\mathrm{fadelholz}$. Ich erwähnte gleich anfangs die Stadtpromenade als den Hauptaufenthaltsort unseres Vogels; diese ist im wahren Sinne des Wortes im englischen Geschmack angelegt: Laubholz ist vorherrschend, Laub- und Nadelholz aller Art wechselt mit offenen Gras- oder Wiesenplätzen oder den herrlichsten Blumenanlagen, bald für sich in Gruppen stehend, bald im bunten Gemisch, bald das Nadelholz, isolirt eingesprengt. So ist es namentlich die Rothtanne, die Weisstanne, die Lärche wie die Weihmuthskiefer, welche oft in 3-5 Stämmen mit einer grösseren Laubholzgruppe vereint, den Promenaden ein so hübsches Ansehen geben, da sie so schön zum hellen Grün des Laubes contrastiren, was recht oft noch erhöht wird, wenn sich das an und für sich unheimliche Laub einer Blutbuche zu ihnen gesellt.

Hier nun, - namentlich wenn diese Busch- und Baumgruppen ein Wenig zurückgezogen sind, so dass ein freies Rasenplätzchen vor ihnen liegt, und vielleicht auch noch ein Teich, und seitwärts sich andere Baumpartien anschliessen, so dass dadurch ein gegen Süden offenes, gegen Norden vielleicht noch durch hohe Rothtannen geschütztes Plätzchen von circa $\frac{1}{2}$ Morg. Grösse entsteht, ist der Girlitz zu Hause; hier sieht man ihn, hier gaukelt er im hellen Sonnenschein über den offenen Platz hin von Baum zu Baum laut singend, und oft fledermaus- oder grünlingsartig flatternd.

Hier nun fand ich das Nest fast stets in dem in einer Laubholzgruppe einzeln eingesprengtem Nadelholz in einer Höhe von 6-20 Fuss; das Nest sitzt am häufigsten auf der Rothtanne, doch fand ich es auch oft genug auf der Weihmuthskiefer, der Weisstanne und Lärche, wie $1 \mathrm{Mal}$ auf dem Taxusbaum und etliche Male auch auf dem Lebensbaum (Frankfurter Kirchhof). Man kann sich denken, dass ich nach Hoffmann's Aufsatz gerade dieser Sache meine ganze Aufmerksamkeit schenkte, und ergab 
sich das 8 jährige Resultat bei circa 40 Nestern von Nadelholz zu Laubholz wie folgt:

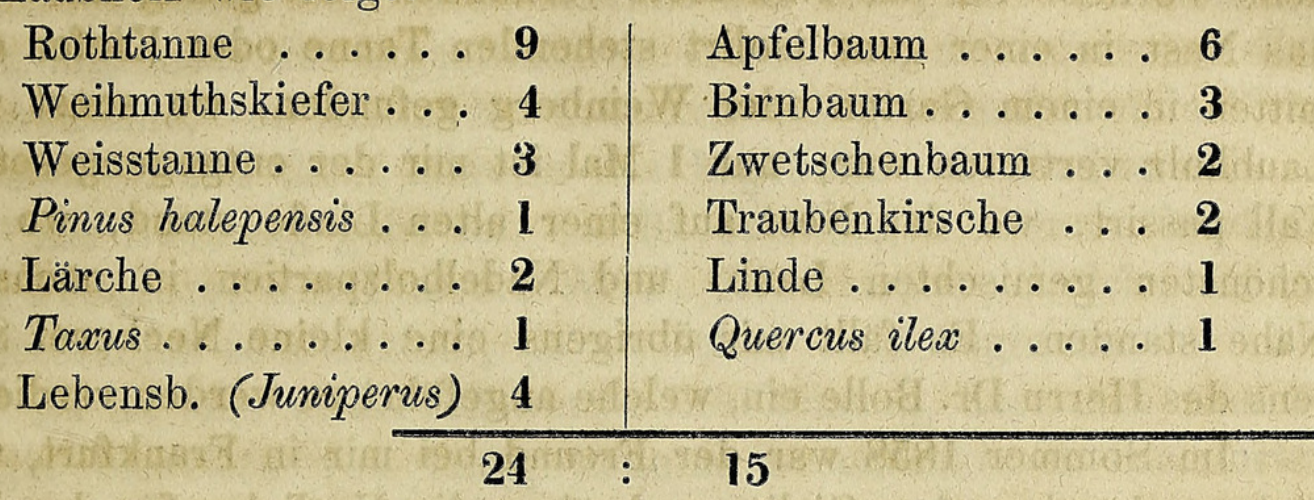

Der Platz des oft sehr variirenden Nestchens ist je nach dem Alter des Baumes verschieden; am liebsten sitzt es bei circa 50 jährigen Rothtannen, auf den vielleicht 15 Fuss hoch entspringenden und his zu 5 Fuss herabhängenden grossen Seitenästen, und zwar gewöhnlich $\mathbf{5}$ Fuss von der abwärts gewendeten Spitze entfernt, zwischen der Hauptrippe selbst und 1-2 Nebenästchen.

Ist der Baum jünger (10-15 Jahr), so steht das Nest gern auf $\frac{2}{3}$ der Höhe neken dem Hauptstamm und der Gabelung zweier Quirläste (ähnlich wie so oft $\mathrm{Fr}$. coelebs bauet). -

Ein Mal fand ich das Nest hängend angebracht, so dass ich es anfänglich für ein Goldhähnchennest hielt, um so mehr, da es in einer alten Rothtanne hing, welche mitten im Laubwald und wenigstens 150 Schritt vom Waldsaum entfernt stand; der Boden des Nestes hatte nicht die geringste Stütze, und waren die Seiten mit 3 senkrecht herabhängenden Zweigen verwebt, kurz, ganz wie beim Goldhähnchen.*)

Im geschlossenen, $d$. h. dichten Nadelwald, kommt der Girlitz meines Wissens nicht vor; sind jedoch Laubbäume eingesprengt, so dass der düstere Charakter gemildert wird, Partien, wie wir sie im westlichen Schwarzwalde finden, so ist es unserm Vogel recht.

Ja selbst in 20jährigen Tannenschonungen, welche mitten im Laubwalde liegen, kommt der Girlitz vor (Forsthaus bei Frankfurt), sie dürfen nur nicht zu dicht, sondern durch Laubbäume und offene Plätze durchbrochen sein. Der Vogel tändelt hier viel über der Nadelholzanlage selbst her, welche durch den hohen sie umstehenden Laubwald geschützt und warm ist, und setzt gern sein Nest am Saum der Schonung in einem Nadelholzbaum selbst.

*) Natürlich ein Ausnahmefall. 
Bei Frankfurt also ist nach meinen Erfahrungen eine wirkliche Vorliebe für das Nadelholz vorhanden; oft genug habe ich das Nest in einer ganz isolirt stehenden Tanne oder Kiefer etc. mitten in einem Garten oder Weinberg gefunden, wo sonst nur Laubholz vertreten war; nur $1 \mathrm{Mal}$ ist mir der entgegengesetzte Fall passirt, wo das Nest auf einer alten Linde stand, wo die schönsten gemischten Laub- und Nadelholzpartien in nächster Nähe standen. Es fällt mir übrigens eine kleine Neckerei Seitens des Herrn Dr. Bolle ein, welche angeführt zu werden verdient:

Im Sommer 1858 war der Freund bei mir in Frankfurt, und viel wurde über den Girlitz und über die Vorliebe für das $\mathrm{Na}$ delholz gesprochen; wir befanden uns gerade im botanischen Garten, als ein Girlitzmännchen vor uns aufflog, sich einige Momente auf einen Fliederstrauch setzte und dann einem grossen Firnissbaum zuflog. „Nun," meinte Bolle, „der Vogel wird sein Nest hier haben und zwar nach Ihrer Theorie oder Praxis im Taxusbaum." "Allerdings,“ meinte ich, „wenn dieser Vogel sich an die alte Frankfurter Regel seiner Kameraden hält, ja." Nun ging es an das Durchsuchen des riesigen Taxus; Bolle schüttelte mit dem Kopf, - wir durchsuchten die Laubbäume, - umsonst, - endlich wieder zurück nach dem Taxus, und - das Nest sass wirklich in ihm. Ich lege auf diesen Fall natürlich kein Gewicht, denn andere ähnliche Fälle stehen ihm zur Seite; aber Freude machte es mir doch.

Schliesslich noch einige Worte über das Nisten auf dem Apfelbaum; Hoffmann will es nicht gern, er hat noch kein Nest gefunden, während in meiner Verhältniss-Rubrik die Zahl 6 steht, und ich beantworte die Sache mit dieser Frage: Wo nistet der Girlitz in jenen Apfelbaumpartien, welche zwischen Frankfurt und den benachbarten Dörfern fast das ganze Feld bedecken, ohne dass gerade andere Baumarten vertreten sind, und wo das Vögelchen so häufig ist?*)

Der Girlitz ist nach verschiedenen Oertlichkeiten seines Aufenthaltes (Frankfurt und Stuttgart) in seinen Lebensverrichtungen und Gewohnheiten bei sonst vielleicht ganz ähnlichen Verhältnissen verschieden, was allein ihn uns zu einem so interessanten Vogel macht, dass er unsere vollkommene Aufmerksamkeit verdient.

") S. Naumannia 1858. p. 146. 
Wohlan denn, ihr Ornithologen des Rheinlandes und anderer girlitzbewohnter Gegenden, schenkt ihm die ganze Aufmerksamkeit, ergründet das Eigenthümliche Eurer Gegend, erforschet alles Interessante, vor Allem die Ursachen des geheimnissvollen Wandertriebes, und theilt hierorts das Ergebniss der Studien mit. -

Als Nachtrag gebe ich die Beobachtungen meines ehrwürdigen Freundes, des Pfarrers W. F. Trinthammer, welche um so interessanter sein dürften, da sie schon 1806 beginnen.

Es ist eine bemerkenswerthe Erscheinung, dass sich manche Vögel bisweilen von langgewohnten beschränkteren Standorten nach gewissen Richtungen hin allmälig weiter verbreiten. Auch an dem Girlitz hat man solch ein langsames Fortrücken von Süden „nach Norden wahrgenommen, und kurz sei Ihnen mitgetheilt, was ich selbst in dieser Hinsicht beobachtet habe. ' Zuvörderst eine Vorbemerkung: Als warmer Freund der Natur hatte mein Vater schon früh in mir ein besonderes Wohlgefallen an dem gesammten gefiederten Völkchen zu wecken gesucht und auch mit dem Girlitz wenigstens par renommée mich bekannt gemacht. Wir wohnten eine Stunde nördlich von Frankfurt a. M., wo ich bis 1809 das Gymnasium besuchte, und jener niedliche Vogel im Weichbilde der Kaiserstadt, Gott weiss, seit wann her, ein freies Beisassenrecht nebst beliebigem Gartenvergnügen genoss. Da Letzteres im naturgeschichtlichen Unterrichte zu Haus bereits öfters erwähnt worden war, so konnte es wahrlich nicht fehlen, dass "unser Frankfurter Vögelchen“, wie meine Schulkameraden gemeinlich ihn nannten, desto stärker mich anziehen musste, weil ich dasselbe nie zuvor in der heimischen Gemarkung gesehen hatte, jetzt aber es überall in den diesseits gelegenen Gärten und noch viel häufiger in den Sachsenhäuser Baumstücken am Mühlberge vor Augen bekam. So wurde denn sechsmal von mir anf's freudigste der Girlitz begrïsst, wenn er im Laufe der Ostermessen zur lustigen Feier des Nickelchestages sich einstellte, wobei nicht vergessen werden darf, dass seine Vermehrung von Jahr zu Jahr zu wachsen schien, nachdem die seit 1806 geschleiften Festungswerke Frankfurts sich nach und nach in herrliche Anlagen umgewandelt hatten. 1818 kehrte ich in das elterliche Haus zurück und lebte dort bis 1835, während welcher Zeit mein Frankfurter Liebling mir etliche Mal von einem Bornheimer Vogelfänger, der ihn am Röderberge gefangen hatte, für meine Stubenvolière geliefert ward. 
Im Sommer 1829 wurde mir zum ersten Mal das Vergnügen zu Theil, in meinem Garten 2 junge Vögelchen an einem Kressenbeet $\mathrm{zu}$ sehen und ihrer habhaft zu werden. Sie mussten wohl in der Nähe ausgebrütet worden sein, denn in den folgenden Jahren liessen sich hin und wieder um das Dorf her Standvögel hören, ohne dass ich jedoch ihre Nester ausfindig machen konnte. Seit 1835 ist Hanau mein Wohnort, wo ich schon früher von 1813 bis 1818 mich aufgehalten habe. Während jener Zeit ist mir nie ein Girlitz hier vorgekommen, - nach meinem 2. Einzuge aber gewahrte ich bald, dass mittlerweile rings um unsere Stadt, also 4 Meilen östlich von Frankfurt, eine ziemliche Ansiedlung stattgefunden hatte, und seit einigen Sommern zeigte sich regelmässig auch in meinem kleinen Hausgarten ein Pärchen, das, da sich in der nächsten Nachbarschaft die günstigste Brutgelegenheit darbietet, sich ansässig gemacht haben muss. Auf Nadelholz soll der Girlitz am liebsten nisten. Hieraus erklärt sich wohl, warum es ihm zu Frankfurt, dessen Gärten mit allerlei Nadelholzarten von jeher geziert waren, so vorzugsweise behagt hat." Glogau, den 24. Februar 1862.

\section{Noch eiwas über den Girlitz.}

Von

\section{Dr. Carl Bolle.}

Im Anschluss an den vorstehenden Aufsatz meines Freundes, des Lieutenant A. von Homeyer, bemerke ich noch Folgendes:

Der Girlitz ist bei Frankfurt so lange einheimisch, als wir überhaupt eine ornithologische Litteratur haben, d. h. seit wenigstens drei Jahrhunderten. Konrad Gessner, dem Aldrovandi fast wörtlich nachschreibt, kennt ihn bereits daselbst unter der noch jetzt von uns gebrauchten Benennung. Er fügt hinzu, der eigentliche deutsche Name sei Fademle, in der Schweiz Schwadeile, im Elsass Gyrle. Auch des Ausdruckes Hirngryll geschieht bei jenen älteren Autoren bereits Erwähnung. „Zu derselben Art gehören nicht minder die Scartzerini genannten Vögelchen, die bei Trient (in Welschtyrol) gefangen, und nach Deutschland gebracht werden. Dort heissen sie „Hirngryllen“, ihres beständigen Singens wegen, welches sie, gleich den Grillen auf sonnigen Aeckern oder den Heimchen um warme Bäder und Backöfen herum, unaufhörlich 


\section{$2 \mathrm{BHL}$ Biodiversity Heritage Library}

Homeyer, Alexander von. 1862. "Zur Naturgeschichte des Girlitz, Fringilla Serinus Lin." Journal

$f u$

r Ornithologie 10, 97-106. https://doi.org/10.1007/bf02005360.

View This Item Online: https://www.biodiversitylibrary.org/item/101707

DOI: https://doi.org/10.1007/bf02005360

Permalink: https://www.biodiversitylibrary.org/partpdf/141248

\section{Holding Institution}

Smithsonian Libraries

\section{Sponsored by}

Biodiversity Heritage Library

\section{Copyright \& Reuse}

Copyright Status: Public domain. The BHL considers that this work is no longer under copyright protection.

This document was created from content at the Biodiversity Heritage Library, the world's largest open access digital library for biodiversity literature and archives. Visit BHL at https://www.biodiversitylibrary.org. 